\title{
Reliability estimation of the sheet stamping process using support vector machines
}

\author{
Jorge E. Hurtado* \\ Universidad Nacional de Colombia, Manizales, Colombia \\ Fax:+5768863220Ｅ-mail: jhurtado14@epm.net.co \\ ${ }^{*}$ Corresponding author
}

\section{Francisco Zárate and Eugenio Oñate}

International Centre for Numerical Methods in Engineering, Technical University of Catalonia, Barcelona, Spain

\author{
E-mail: zarate@cimne.upc.es_E-mail: onate@cimne.upc.es
}

\begin{abstract}
An important concern in sheet stamping is the risk of obtaining brittle final products that can be affected by fracture. Monte Carlo simulations presented herein show that this is governed by two main factors, namely static and dynamic friction coefficients. Whereas the latter correlates in a non-linear manner with minimum and maximum end thickness, the relationship of these design parameters to the former exhibits a bifurcation that is typical of highly non-linear phenomena, in which there is a sensitivity to small perturbations of the input values (chaos). In order to estimate the reliability of the process (i.e., the probability of obtaining brittle products due to low minimum and maximum thicknesses) with a reduced number of Monte Carlo runs, it is proposed to assimilate the problem to a pattern recognition task, due to the existence of two classes, namely robust and brittle. Among many pattern recognition algorithm that are useful to this end, use is made of support vector machines, as this incorporates the powerful tool of class margins that allow a drastic reduction of the number of simulations.
\end{abstract}

Keywords: sheet stamping; pattern recognition; artificial intelligence; support vector machines; Monte Carlo simulation.

Reference to this paper should be made as follows: Hurtado, J.E., Zárate, F. and Oñate, E. (2005) 'Reliability estimation of the sheet stamping process using support vector machines', Int. J. Vehicle Design, Vol. 39, Nos. 1/2, pp.110-124.

Biographical notes: Civil Engineer by the National University of Colombia; Master in Earthquake Engineering and Doctor in Civil Engineering by the Technical University of Catalonia (Spain). His theoretical research areas include nonlinear systems, stochastic systems, Monte Carlo simulation, stochastic mechanics, statistical learning and artificial intelligence applications to nonlinear systems. His applied research fields are material modeling, earthquake engineering and natural disasters. The results of these research activities have been published in leading journals like Structural Safety, Probabilistic Engineering Mechanics, Computer Methods in Applied Mechanics and Engineering, Journal of Structural Engineering, Archives of Computational Methods in Engineering, among others, as well as in numerous Conference Proceedings. He is also the author of the book Structural Reliability - Statistical Learning Perspectives (Springer Verlag, 2004). 
Civil Engineer, PhD by the Universitat Politècnica de Catalunya (UPC) (1992). $\mathrm{He}$ has wide experience in the development and application of finite element methods for structuraldynamic analysis. He has developed rotation free shell triangles for analysis of large scale shell problems in civil, mechanical and manufacturing problems, among others. He has also good experience in developing tools for distributed computing using grid technology. He is the author of more than 20 papers in computational mechanics.

Civil Engineer, $\mathrm{PhD}$ by the University of Swansee. Director of the Internactional Center for Numerical Methos in Engineering (CIMNE). President of the International Association for Computational Mechanics (IACM) (from 2002), and President of the European Community on Computational Methods in Applied Sciences (ECCOMAS) (from 2000).

Author of a text book on finite element structural analysis, editor of 30 books and author of more than 120 research papers on development and applications of numerical methods (finite elements, finite volumes and meshless method) to structural and fluid dynamics problems and the development of decision-support system in engineering.

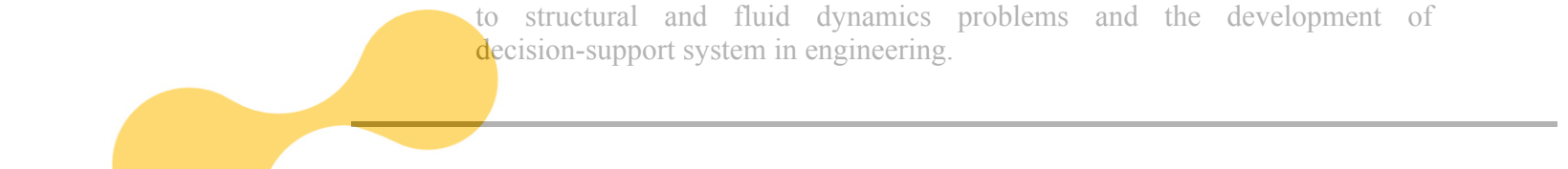

\section{Introduction}
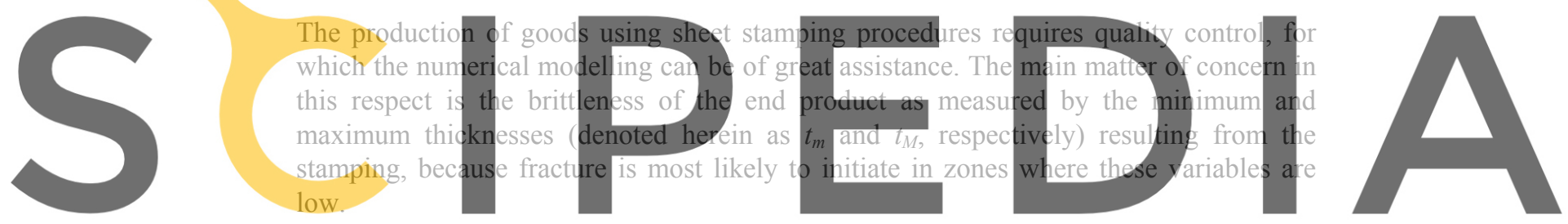

Friction plays an important role in sheet metal forming process and is therefore of

Register for free majorimprtance for reliabla fininte element simulations of these progesses. Fitr these the watermark

coefficient is constant parameter. From a wider point of view, this is not satisfactory

because the friction coefficient depends on local contact conditions like pressure, combined surface roughness of the sheet and the tools, lubricant viscosity, and velocity of the surfaces.

During sheet forming process, the friction between sheet and tools has an active role in affecting the material flow, the strain distribution, and the forming force. It also takes part in determining the forming failures (e.g., wrinkling, tearing, and surface distortion) and the location of these failures. Hence, an accurate simulation of the sheet forming process requires a detailed understanding of frictional behaviour under actual forming condition. Since the frictional force is known to be a complicated function of material properties and process parameters, it is unrealistic to use a single test to accurately represent the sheet metal forming process.

In the frictional problem, two cases can be distinguished. At the early stage of the process where a full stick condition between the sheet and tools is verified, a tangential force appears opposing to the relative slip. At this phase, the active friction coefficient is the so-called static one $\left(\mu_{\mathrm{s}}\right)$ Once a threshold value in the modulus of the force is reached, a slip condition is verified; hence, the dynamic friction coefficient $\left(\mu_{d}\right)$ becomes dominating. 
Numerical simulations using random values of these two parameters (i.e., Monte Carlo simulations) show that the sheet stamping problem exhibits a chaos-like phenomenon, as small perturbation of the static friction coefficient, when the dynamic coefficient is relatively large, lead to either a small or a large value of the end thicknesses. This phenomenon is known as bifurcation in the theory of non-linear systems. Obviously, this is caused in this case by the high non-linear nature of the stamping process.

This implies that the probability of having brittle products should be estimated by a Monte Carlo simulation involving the following steps:

- the probabilistic description of the two friction coefficients, as the main stochastic variables determining the appearance of brittle products (other random variables and fields such as the elastic moduli have not been found to be of importance in this respect in the numerical simulations carried out in the study reported herein);

the use of an updated Lagrangian formulation for the finite element problem of each run;

- the definition of a failure criterion, which could be the exceedance of certain thresholds for the minimum and maximum end thicknesses.

The reliability $R$ of the product would be the number of Monte Carlo samples on the safe

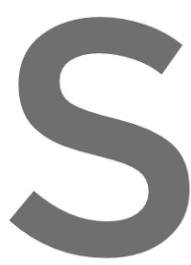

zone divided

$P_{f}=1-R$

Despite the

costly in computat

failure requires

this implies
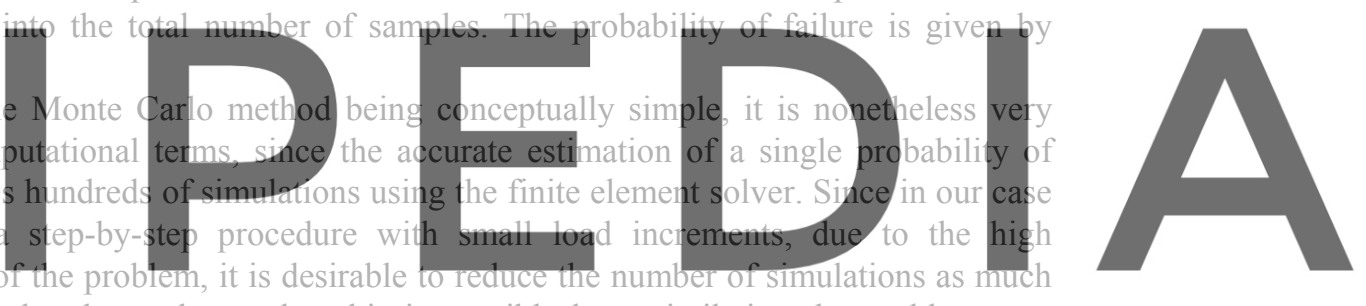

as possible. It has been shown that this is possible by assimilating the problem to a

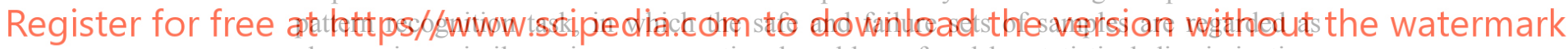

classes, in a similar vein as conventional problems faced by statistical discrimination,

such as plant or disease classification (Hurtado and Alvarez, 2001, 2003). The calculation of pattern recognition methods on the basis of some training samples yields a discriminating function, which can henceforth be used to assign new incoming samples to one or to the other class. Therefore, it is expected that they can be used as a substitute of the finite element solver for most of the samples needed in the estimation of the sheet stamping reliability.

On the basis of such an approach, a manifold of pattern recognition methods are offered to the analyst. In fact, more than ten classification methods are described in the handbook (Hastie et al., 2001), which include Bayesian and kernel methods, classification trees, neural networks, etc. The selection of a single method among them is hindered by the so-called no-free-lunch theorem, which roughly speaking states that there are no reasons to prefer one classification method over other (Duda et al., 2001). However, in the present case, there is a special circumstance that facilitates the selection. A major difference of Monte Carlo samples with respect to those normally used in statistical analyses is that they are synthetic, in the sense that they are produced by computer codes and not drawn from nature. As a consequence, it is not necessary that they be available before initiating the calculation of the discriminating function, as they can be produced in the calculation process. Among pattern recognition methods, this 
important feature of synthetic samples is best exploited by an artificial intelligence method known as support vector machines (Vapnik, 1998) because, in addition to the discriminating function as such, they include two ancillary functions known as margins, which can be used to reduce the search domain of the sample space, thus subjecting to control the generation of new training samples. A method in that sense has recently been proposed by the first author (Hurtado and Alvarez (2003). The method can be considered as a member of the family of controlled Monte Carlo simulation techniques, such as those developed for solving random vibration problems (Pradlwarter and Schuëller, 1999). See Hurtado (2004) for a thorough analysis on the applicability of pattern recognition techniques in structural mechanics.

According to the above said, the present paper has the following purposes:

- to illustrate the bifurcation problems posed by the random friction coefficients on the sheet stamping process and its implications on the product reliability and quality control;

to show how this reliability can be economically computed using the support vector method of pattern recognition, because it yields a discriminating function that can be used as a substitute of the finite element solver for most of the samples needed in its estimation.

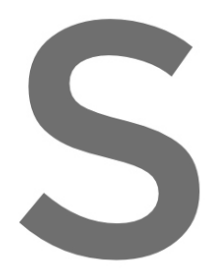

The rest of paper is divided into four sections as follows. First, some backgrounds on the finite element approach employed to the The final section is devo

2 Backgrounds
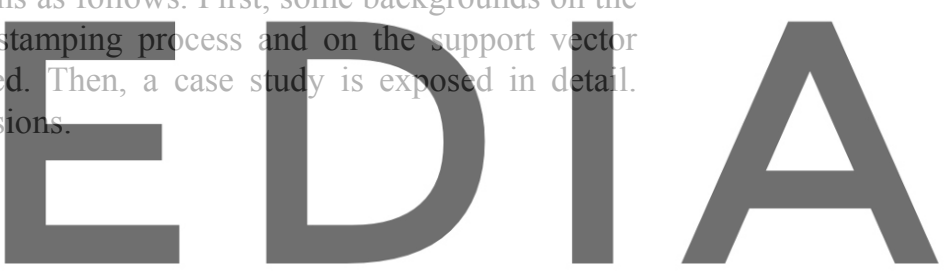

\section{Register for free at ht tinite element approgich}

This section summarises the main concepts and equations involved in the mechanical analysis of a stamping problem, as it is needed for a more complete description of the study reported herein.

As is well known, this mechanical problem must be dealt with in both Eulerian (or spatial) and Lagrangian (or material) coordinate system, denoted as $X$ and $x$, respectively, in the sequel. The displacement field is defined either in the material or spatial systems as

$$
\begin{aligned}
& X=X+u(X, t) \\
& X=X-u(X, \mathrm{t})
\end{aligned}
$$

and the deformation gradient tensor is defined in tensor notation as

$$
F_{i K}=\frac{\partial x_{i}}{\partial X_{K}}
$$

In terms of the tensor $F$, the left and right Cauchy-Green tensors, $P$ and $Q$, are defined as

$$
P_{i j}^{-1}=X_{K, i} X_{K, j}
$$




$$
Q_{K L}=x_{i, K} x_{i, L}
$$

These tensors allow the definition of the Green-Lagrange $E$ and the Almansi $\in$ strain tensors as

$$
\begin{aligned}
& E=(Q-I) \\
& \in=\frac{1}{2}\left(I-P^{-1}\right)
\end{aligned}
$$

In analysing a stamping problem, it is necessary to consider both the equation of motion in spatial coordinates and material coordinates. The first one reads

$$
\nabla x \sigma+\rho b=\rho a \quad x \in \Omega^{t}
$$

with boundary and initial conditions, given by,

$$
\begin{aligned}
& n \times \sigma=\mathrm{t} \quad x \in \Gamma^{t} \\
& u=u_{0} \quad v=v_{0} \quad x \in \Omega^{t}
\end{aligned}
$$

respectively.

In material coordinates, the equation of motion is
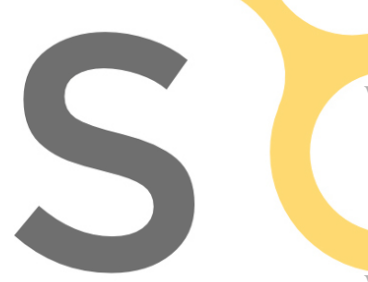

\section{$\nabla \times T+$}

with the following boundary and initial conditions: $N \times T=1$
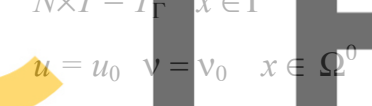

where $T$ is the first stress tensor of Piola-Kirchoff and
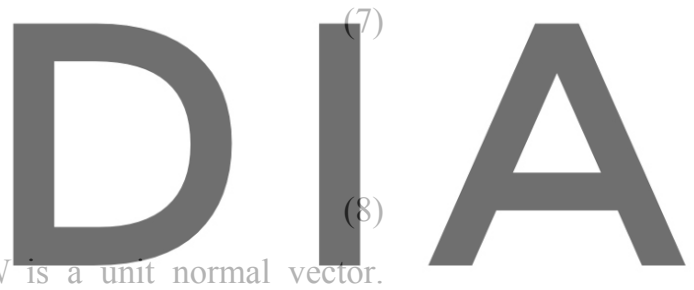

The weak form of the equilibrium equations is

Register for free at https//www.scipedia.com to download the version without the watermark

$$
\begin{aligned}
& \int_{\Omega^{D}} T: \delta F \cdot d \Omega=\int_{\Omega^{D}} \rho_{0}\left(\mathrm{~b}_{0}-A\right) \delta u \cdot d \Omega+\int_{\Gamma^{D}} T \delta u \cdot d \Omega \\
& \int_{\Omega^{D}} S: \nabla \times \delta E \cdot d \Omega=\int_{\Omega^{D}} \rho_{0}\left(b_{0}-A\right) \delta u \cdot d \Omega+\int_{\Gamma^{D}} T \delta u \cdot d \Omega
\end{aligned}
$$

where $T$ and $S$ are the first and second Piola-Kirchoff tensors, respectively, $F$ is the gradient tensor, $E$ is the Green-Lagrange tensor, and $\in$ the Almansi strain tensor. The constitutive equations are those of a hypoelastic material (Belytschko et al., 2000). The form of the constitutive tensor depends on the modelling of the material as anisotropic, orthotropic, or isotropic.

Using an updated Lagrangian formulation, the first of the equations (10), written in matrix form, is

$$
\int_{\Omega^{t}} \delta u^{T} \rho \ddot{u} \cdot d \Omega+\int_{\Omega^{t}} \delta \in^{T} \cdot \sigma \cdot d \Omega-\int_{\Omega^{t}} \delta u^{T} \cdot \rho b d \Omega-\int_{\Gamma^{t}} \delta u^{T} \cdot d \Omega=0
$$

where 


$$
\begin{aligned}
& \sigma=\left[\begin{array}{llllll}
\sigma_{11} & \sigma_{22} & \sigma_{33} & \sigma_{12} & \sigma_{13} & \sigma_{23}
\end{array}\right]^{T} \\
& \in=\left[\in_{11} \in_{22} \in_{33} \in_{12} \in_{13} \in_{23}\right]^{T}
\end{aligned}
$$

where the conventional elasticity definitions of the components of $\in$ are applied. Supposing that the kinematics of body is compatible with the displacement field, the following finite element discretisation is adopted:

$$
u(X)=N(X) r
$$

where $\mathrm{N}(X)$ is the shape function matrix and $r$ is the vector of nodal displacements. The first variation of the displacement field and that of the Green-Lagrange tensor are

$$
\delta u(X)=N(X) \delta r \delta \in(\mathrm{X})=B(\mathrm{X}) \delta r
$$

where $B$ is the deformation matrix, given in this case by

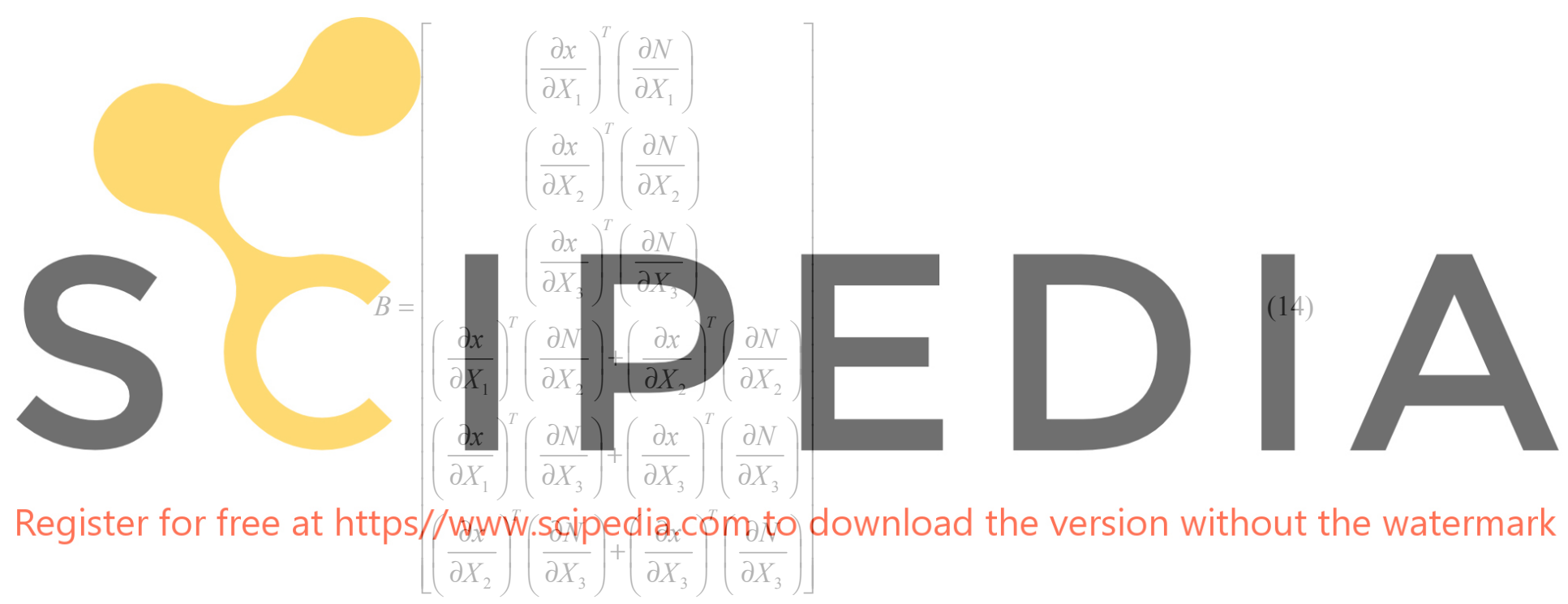

The application of this discretisation leads eventually to a matrix differential equation of the form

$$
M \ddot{r}+F^{\text {int }}-F^{\text {ext }}=0
$$

where the matrices $M, F^{\mathrm{int}}$, and $F^{\mathrm{ext}}$ result from the assemblage of the element matrices

$$
\begin{aligned}
& M^{(e)}=\int_{\Omega^{e}} \rho N^{T} N d \Omega \\
& F^{\mathrm{int},(e)}=\int_{\Omega^{e}} B^{T} \sigma d \Omega \\
& F^{\mathrm{ext},(e)}=\int_{\Omega^{e}} N^{T} \rho b d \Omega+\int_{\Gamma^{e}} N^{T} t d \Gamma
\end{aligned}
$$

Since the mass matrix appearing in the above differential equation is not diagonal, thus making the solution of the problem cumbersome, a modification of the system is in order. To this purpose, an equivalent solution of the form 


$$
M^{*} \ddot{r}+C \dot{r}+F^{\mathrm{int}}+F-F^{\mathrm{ext}}=0
$$

where $M^{*}$ is a diagonal mass matrix and $C$ is a damping matrix, which is introduced in order to reduce the vibrations. The term $F^{\text {cont }}$ is introduced to represent the contact force.

\subsection{Support vector machines}

In this paragraph, the support vector method for pattern recognition is summarised after Vapnik (1998).

Let us consider two classes that are linearly separable. Let the patterns be $\left\{x_{1}, x_{2}, \ldots, x_{n}\right\}$ and the classes to which they belong be identified as $c_{i} \in\{-1,1\}$ $i=1,2, \ldots, n$. A separating hyperplane of the form

$$
g(x)=\langle w \cdot x\rangle+b=0
$$

is sought, where $w$ is a vector of parameters that define the normal vector to the hyperplane, and $b$ is the threshold. The condition imposed to this hyperplane is that it maximises the distance to the given patterns, as this allows the best confidence on the classification it performs. The optimisation problem then reads

$$
\max _{w} \min _{i}\left\{\left\|x-x_{i}\right\|:(w \cdot x)+b=0, i=1,2, \ldots, n\right\}
$$
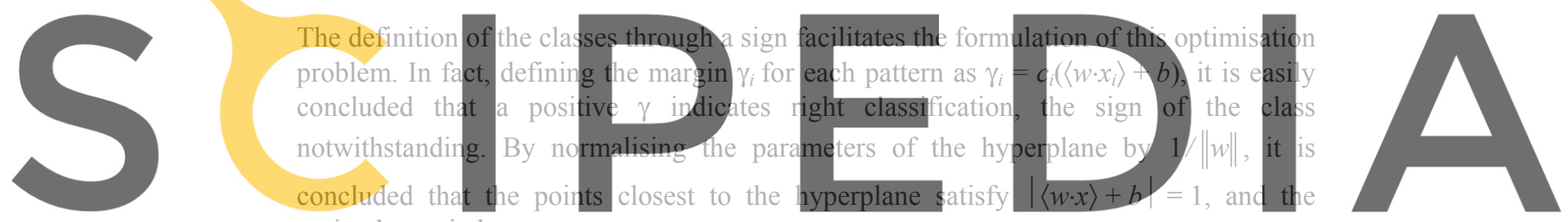

\section{Register for free at https/fwww.scipedia.com to download the version witho(lt) the watermark}

as can be easily demonstrated. The optimisation problem becomes

$$
\begin{aligned}
& \text { minimise } \Omega(w)=\frac{\|w\|^{2}}{2} \\
& \text { subject to: } c_{i}(\langle w \cdot x\rangle+b) \geq 1, \quad i=1,2, \ldots, n
\end{aligned}
$$

which can be recast as

$$
L(w, b, \alpha)=\frac{1}{2}\|w\|^{2}-\sum_{i=1}^{n} \alpha_{i}\left[c_{\mathrm{i}}\left(\left\langle w \cdot x_{i}\right\rangle+b\right)-1\right]
$$

via Lagrange multipliers $\alpha_{i} \geq 0$. The problem is hence to find a saddlepoint because it is necessary to minimise the loss function with respect to the hyperplane parameters $(w, b)$, while maximising it with respect to the Lagrange multipliers. The solution is

$$
w=\sum_{i=1}^{n} \alpha_{i} c_{i} x_{i}
$$




$$
\sum_{i=1}^{n} \alpha_{i} c_{i}=0
$$

Taking into account the positiveness of the Lagrange multipliers set forth above, equation (22) has a very important meaning for the structural safety problem: the separating hyperplane can be expressed only in terms of the patterns that have a positive Lagrange multipliers, while the rest are no longer needed. These special patterns are the support vectors, which have the special property of lying just on the margins defined above, as it can be easily demonstrated by invoking the well known Karush-Kuhn-Tucker complementarity condition of optimisation theory (Kall and Wallace, 1995): $\left[c_{i}\left(\left\langle w x_{i}\right\rangle+b\right)-1\right]=0, I=1,2, \ldots n$. This interpretation is crucial for the controlled Monte Carlo algorithm proposed herein, since it implies that the only samples needed to update the classifier are those lying in the band defined by the margins.

The above means that the solution for the weights of the hyperplane can be expressed as a convex combination of some input patterns in the form

where $s$ is the number of support vectors. An interpretation of this equation is that the optimal hyperplane can be defined only in terms of the patterns that are the closest to it,

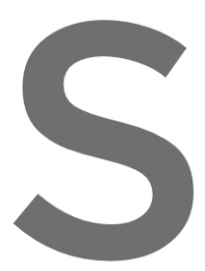

which agrees with intuition.

For passing into the non-linear separation, it is convenient to cast the optimisation problem in terms of the done by replacing equations

Maximise $\mathrm{v}(\alpha)=\sum_{i=1}^{n} \alpha_{i}-\frac{1}{2} \sum_{i=1}^{n} \sum_{i=1}^{n} \alpha_{i} c$
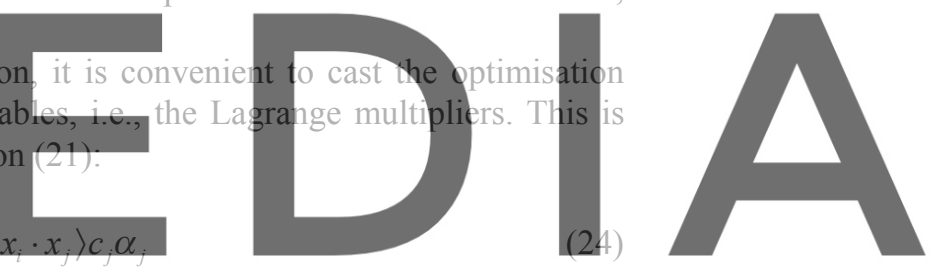

Register for free at https//www.scipedia.com to download the version without the watermark

$$
\sum_{i=1}^{n} \alpha_{i} c_{i}=0
$$

The solution of this quadratic optimisation problem should be substituted into equation (23) to obtain the values of the hyperplane parameters. The threshold $b$ can then be obtained from the Karush-Kuhn-Tucker condition. The classification function then reads

$$
c(x)=\operatorname{sgn}(g(x))=\operatorname{sgn}\left(\sum_{i=1}^{n} \alpha_{i} c_{i}(\langle w \cdot x\rangle)+b\right)
$$

For non-linear separation, it is very important to remark that a characterising feature of hyperplane discrimination is that the probability of correct classification of $n$ samples increases with the number of dimensions $d$ (Fine, 1999). In other words, increasing the dimension of the problem by projecting the samples onto a higher dimensional space, while maintaining a hyperplane formulation, is a good means for assuring correct classification of the training set. This is easily done by generalising the last equation to 


$$
c(x)=\operatorname{sgn}(g(x))=\operatorname{sgn}\left(\sum_{i=1}^{n} \alpha_{i} c_{i}\left(K\left(x_{i}, x\right)+b\right)\right)
$$

where the linear kernel in equation (26) has been substituted by the general kernel

$$
K(x, z)=\langle\phi(x) \ldots \phi(z)\rangle
$$

in which function $\varphi(\cdot)$ represents the non-linear mapping. The interesting point in this kernel presentation is that the non-linear mapping $\varphi(\cdot)$ need not be known explicitly. This allows constructing positive-definite kernels with virtually infinite dimensions in a Hilbert space using the spectral decomposition granted by Mercer's theorem (Cristianini and Shawe-Taylor, 2000). Such a large dimensionality implies the highest probability of correct classification using a hyperplane, according to the above said. Table 1 shows some kernels widely applied in support vector classification.
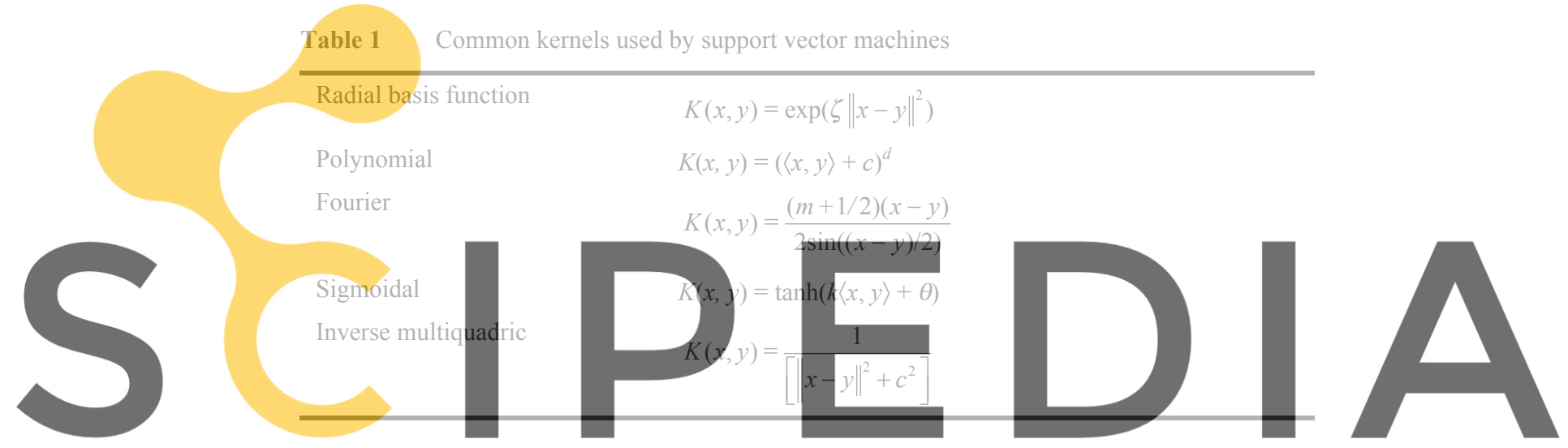

\section{Register for free at hetess $\$$ wwdww.scipedia.com to download the version without the watermark}

\subsection{Problem description}

The square cup deep drawing problem is one of the most practical industrial application of sheet stamping. This problem has been the subject of much research and represents a benchmark test that was proposed by the Numisheet' 93 .

The benchmark test reported herein features the ability to simulate a large amount of material draw-in with a nearly vertical wall and the ability to efficiently treating the frictional contact. It consists of the analysis of a deep drawing process of a square sheet into a square cup. The geometries of the punch, die, and blankholder are shown in Figure 1. For this deep drawing problem, the punch travel is $40 \mathrm{~mm}$. 
Figure 1 Geometry of the square cup deep drawing problem

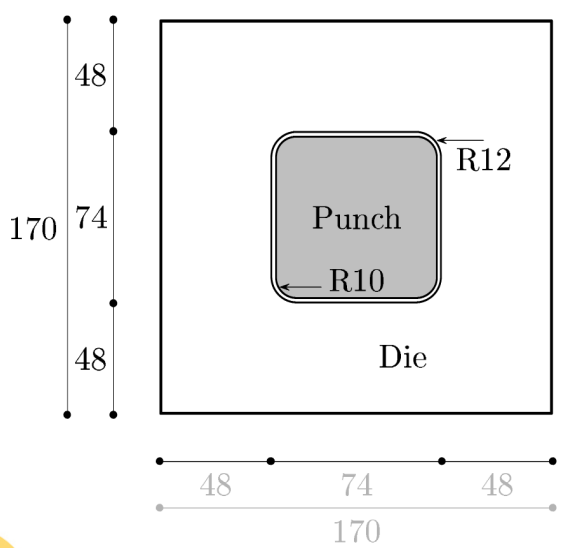

$(a)$

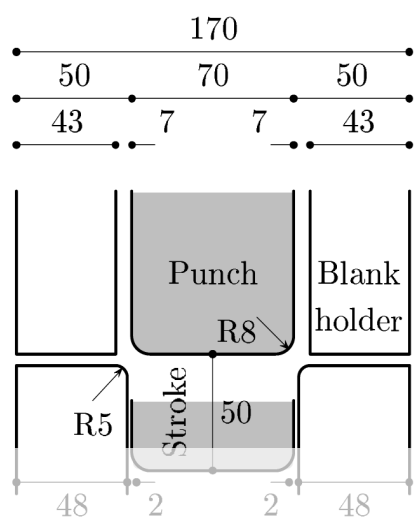

(b)

The sheet was modelled using triangular shell layered elements. The blankholder, punch, and die were modelled using rigid elements. The initial geometry of the tools as well as the sheet geometry after deformations were complete in a single simulation and are shown in Figures 2 and 3. The sheet material is mild steel using a non-linear hardening elasto-plastic model with the following properties: Young's modulus E $=206 \mathrm{GPa}$, Poisson's ratio $v=0.3$, mass density $\rho=7800 \mathrm{~kg} / \mathrm{m}^{3}$, and the initial yield value $C=565.3 \mathrm{MP}$. The blankholder naterial was steel using a linear elastic nodel with the following properties: Young's modulus $E=210 \mathrm{GPa}$, Poisson's ratio $v=0.3$, and mass density $\rho=7800 \mathrm{~kg} / \mathrm{m}^{3}$. The die was held in $\mathrm{pl}$ Contact pair sturfaces between the tools an sheet die sheet, and blankholder

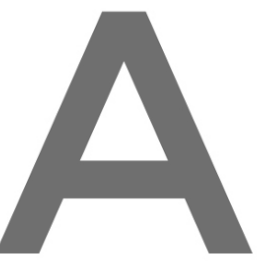
contact pairs were modelled using a constant friction coefficient.

Register for free at https//www.scipedia.com to download the version without the watermark Figure 2 Initial geometry of the square cup deep drawing problem

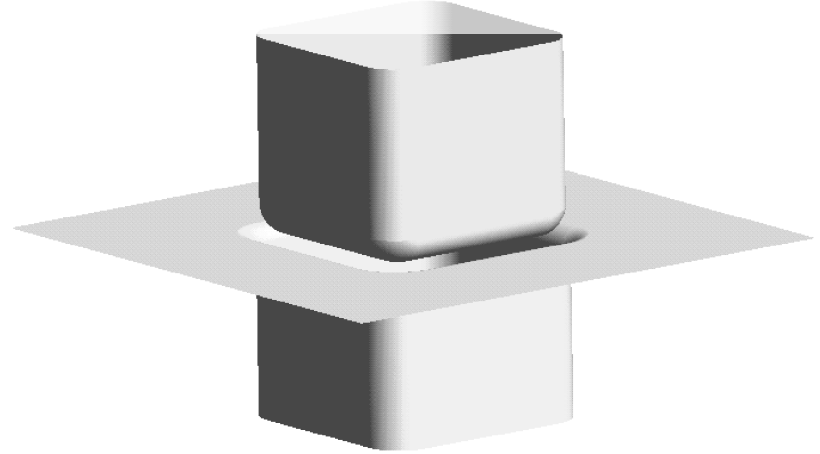


Figure 3 Final deformed shape

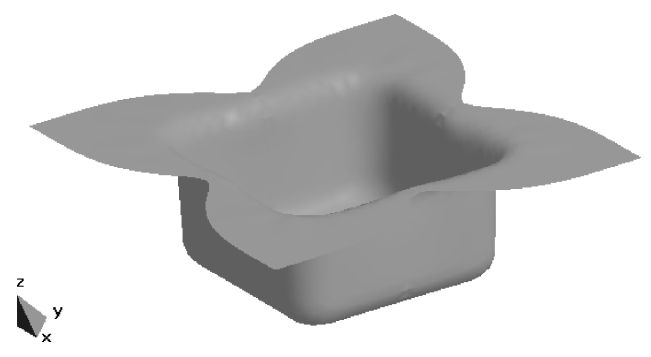

A constant blankholding force of $19.6 \mathrm{kN}$ was applied to the surface of the blankholder as a surface load on those elements. The punch was moved vertically for $40 \mathrm{~mm}$ by applying a sinusoidal velocity over the total analysis time period. The total response time was obtained using an automatic time stepping calculation and was terminated when the punch stroke was completed. Material non-linearities were considered to be rate independent.

A crucial stage in any probabilistic problem is the selection of the random variables together with their probability density functions. In the present case, the static and dynamic friction coefficients were selected as the only input random variables on the basis of previous experience. Since knowledge of the probabilistic information regarding these variables is rather scarce, it was assumed that they obey a lognormal distribution, as this assures a positive value. For both coefficients, the mean and standard deviation were selected as 0.15 and 0.03 , respectively. These values were applied on the contact surface pair sheet-punch. The observed output random variables were the minimum and maximum thicknesses of the sheet after the forming process.

Two codes developed at the International Centre for Numerical Methods in Engineering of Barcelona were used for the calculations: the finite element code STAMPACK for the sheet stamping problem and the code STAC for the Monte Carlo analyses. The STAMPACK code uses the new finite elements described in Oñate et al. (1996, 1999). The support vector problem, which determines the new samples to be generated in the controlled Monte Carlo simulation, was solved with a special code developed for such purpose.

\subsection{Monte Carlo analysis}

A first analysis conducted on this example was a conventional Monte Carlo simulation, intended to illustrate the bifurcation problems arising from the highly non-linear behaviour of the stamping process. The number of Monte Carlo samples was 300 .

Figure 4 shows the relationship between the minimum end thickness and the static friction coefficient. It can be observed that a bifurcation of the cloud of points occurs for values of the coefficient around the mean. The low values of the end minimum thickness are associated also to the upper tail values of the dynamic friction coefficient, as shown in Figure 5. Similar figures were obtained for the relationships of both friction coefficients to the maximum end thickness. All this means that the risk of forming sheets likely to suffer fracture corresponds to static coefficients around the nominal value used commonly in deterministic calculations and to somewhat large values of the dynamic parameter. 
Figure 4 Relationship between the minimum end thickness and the static friction coefficient

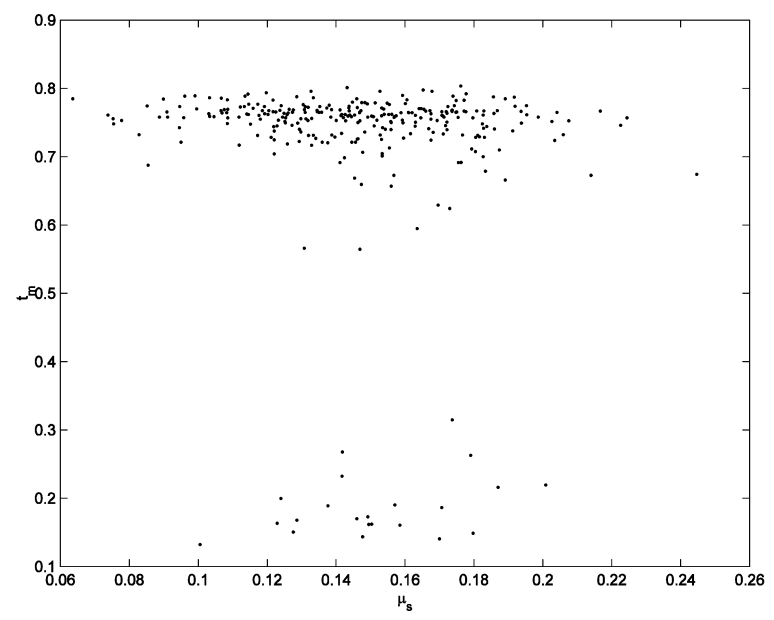

Figure 5 Relationship between the minimum end thickness and the dynamic friction coefficient

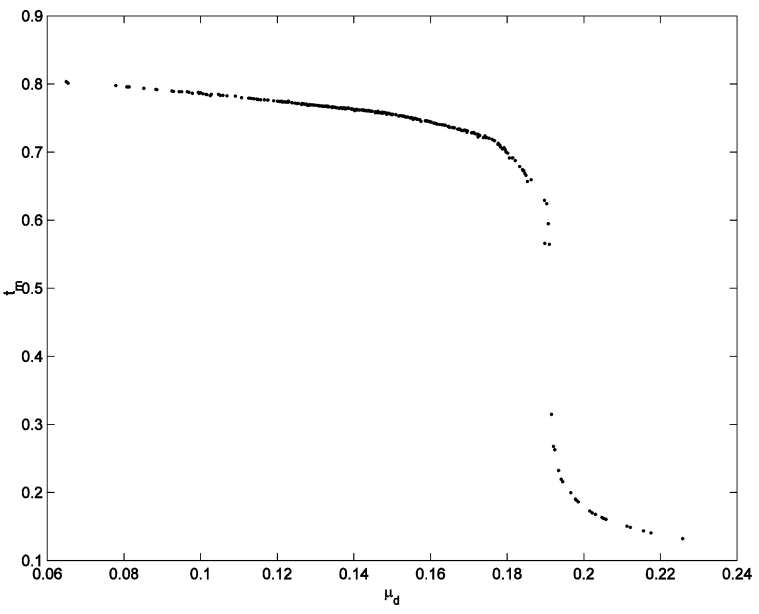

\subsection{Reliability classification analysis}

Figure 6 shows that the minimum and maximum end thicknesses are intimately related. Thus, a product can be specified as brittle when these values are lower than certain thresholds. Assuming that the failure domain is defined as $t_{m}<0.5 \mathrm{~m}$ and $t_{M}<1.4 \mathrm{~mm}$ for this example, the above Monte Carlo simulation shows that the probability of failure is $P_{f}=22 / 300=0.0733$. However, 300 samples are required to estimate this figure with such a good accuracy. It is shown in this paragraph that the computational cost is much lower when using the Monte Carlo method controlled by support vector machines in the following manner: Two simulations of pairs $\left(\mu_{s}, \mu_{d}\right)$, corresponding to the safe and unsafe zones, are first produced. This can be easily done by simply assigning a low and a large value of the dynamic friction coefficient, since this is tightly related to the end thickness as shown above. Next, a support vector classifier is calculated and the resulting margin functions are used to generate a new sample inside the margin band, because 
samples out of that zone produce no improvement of the classifier, according to the Karush-Kuhn-Tucker conditions.

Proceeding in this manner, the classifier is sequentially updated until the margin becomes sufficiently narrow.

Figure 6 Relationship between the minimum and maximum end thicknesses

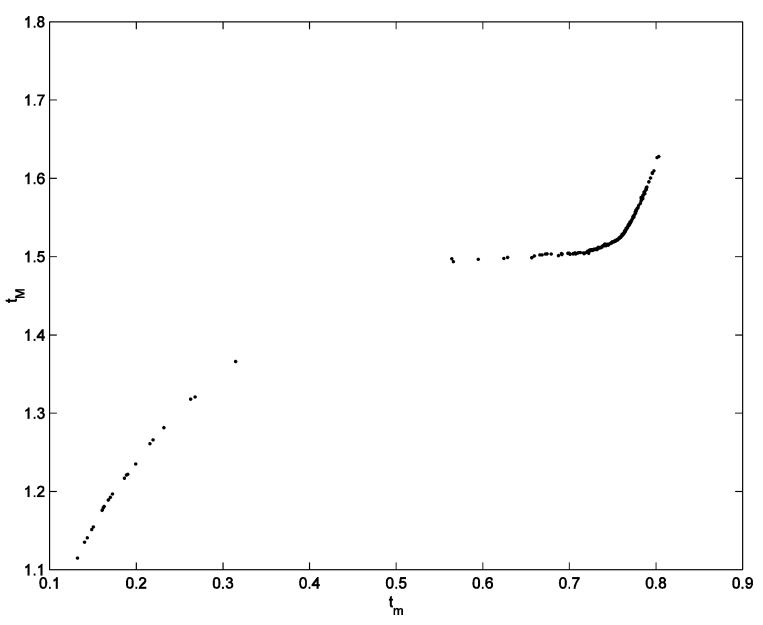

A support vector machine with a polynomial kernel with parameters $c=1, d=3$ (conventional ones) was selected. Figure 7 shows the discrimination and margin functions obtained in this manner. Notice that the end margin band is very narrow and that the 300 samples used in the previous analysis are correctly classified. The training samples $\left(\mu_{s}, \mu_{d}\right)$ were picked up in the above described manner from the databank used for the previous Monte Carlo simulation. Only 15 calls of the finite element solver were required to build the classifier, which evidently yields the same failure probability when used for classifying the rest (285) samples not used in its training.

Figure 7 Support vector discriminating function. The support vectors appear as black circles

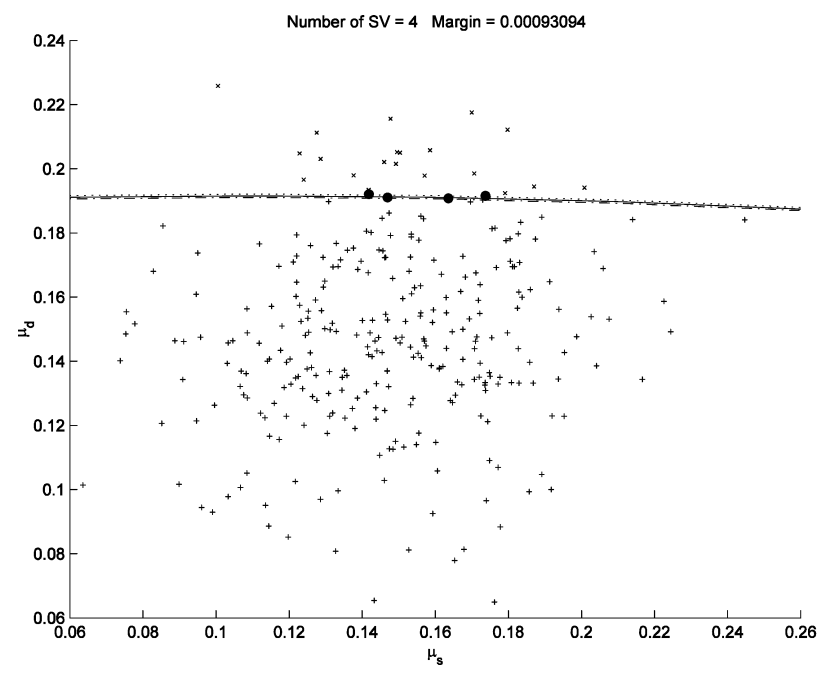




\section{Conclusions}

Monte Carlo simulations of sheet stamping process show that the leading variables in determining the generation of products with a high risk of fracture are the static and dynamic friction coefficients. In particular, a sudden jump from acceptable to unacceptable end thickness arises when the latter parameter surpasses a certain critical value, in combination with static coefficients around the mean. This appears as a bifurcation, which is a phenomenon that is likely to occur in non-linear systems.

A method for calculating the reliability of sheet stamping products has been proposed. It is based on the consideration of the problem as a pattern recognition task. The use of a specific pattern recognition method known as support vector machines over neural networks, Bayesian approaches, and other techniques is justified by the availability of margin functions that allow to devise a controlled Monte Carlo simulation procedure. By means of a numerical example, it has been shown that a reduced number of finite element calculations suffice for creating a discriminating function that can be used with new samples of the friction coefficients for calculating the probability of obtaining brittle products.

\section{Acknowledgements}

Financial supports for the realisation of the present research have been received from the Universidad Nacional de Colombia and the International Centre for Numerical Methods in Engineering of Barcelona. The supports are gratefully acknowledged.

\section{References}

Belytschko, T., Liu, W.K. and Moran, B. (2000) Nonlinear Finite Elements for Continua and Structures', John Wiley and Sons, Chichester.

Cristianini, N. and Shawe-Taylor, J. (2000) An Introduction to Support Vector Machines and Other Kernel-based Methods, Cambridge University Press, Cambridge.

Duda, R.O., Hart, P.E. and Stork, D.G. (2001) Pattern Classification, John Wiley and Sons, New York.

Fine, T. (1999) Feedforward Neural Network Methodology, Springer Verlag, New York.

Hastie, T., Tibshirani, R. and Friedman, J. (2001) The Elements of Statistical Learning, Springer Verlag, New York.

Hurtado, J.E. (2004) Structural Reliability - Statistical Learning Perspectives, Springer Verlag, Heidelberg.

Hurtado, J.E. and Alvarez, D. A. (2001) 'Neural network-based reliability analysis: a comparative study', Computer Methods in Applied Mechanics and Engineering, Vol. 191, pp.113-132.

Hurtado, J.E. and Alvarez, D.A. (2003) 'A classification approach for reliability analysis with stochastic finite element modeling', Journal of Structural Engineering, Vol. 129, pp.1141-1149.

Kall, P. and Wallace, S.W. (1995) Stochastic Programming, John Wiley and Sons, Chichester. 
Oñate, E., Cendoya, P., Rojek, J. and Miquel, J. (1996) 'A simple thin shell triangle with translational degrees of freedom for sheet stamping analysis', $3 \mathrm{rd}$ International Conference on Numerical Simulation of 3D Sheet Forming Processes, Numisheet, Dearbon.

Oñate, E., Zárate, F., Rojek, J., Duffet, G. and Neamtu, L. (1999) 'Advances in rotation free shell elements for sheet stamping analysis', 4th International Conference on Numerical Simulation of $3 D$ Sheet Forming Processes, Numisheet, Besancon.

Pradlwarter, H.J. and Schuëller, G.I. (1999) 'Assessment of low probability events of dynamical systems by controlled Monte Carlo simulation', Probabilistic Engineering Mechanics, Vol. 14, pp.213-227.

Vapnik, V.N. (1998) Statistical Learning Theory, John Wiley and Sons, New York. 